\title{
Preservation of the Classical Meanness Property of Some Graphs Based on Line Graph Operation
}

\author{
G. Muhiuddin $\mathbb{D}^{1},{ }^{1}$ A. M. Alanazi, ${ }^{1}$ A. R. Kannan, ${ }^{2}$ and V. Govindan ${ }^{3}$ \\ ${ }^{1}$ Department of Mathematics, University of Tabuk, Tabuk 71491, Saudi Arabia \\ ${ }^{2}$ Department of Mathematics, Mepco Schlenk Engineering College (Autonomous), Sivakasi 626005, Tamil Nadu, India \\ ${ }^{3}$ Department of Mathematics, Sri Vidya Mandir Arts \& Science College Katteri, Uthangarai, Tamilnadu 636902, India
}

Correspondence should be addressed to G. Muhiuddin; chishtygm@gmail.com

Received 28 July 2020; Revised 4 February 2021; Accepted 26 February 2021; Published 13 March 2021

Academic Editor: Elena Guardo

Copyright (c) $2021 \mathrm{G}$. Muhiuddin et al. This is an open access article distributed under the Creative Commons Attribution License, which permits unrestricted use, distribution, and reproduction in any medium, provided the original work is properly cited.

In the present paper, we introduce the classical mean labeling of graphs and investigate their related properties. Moreover, it is obtained that the line graph operation preserves the classical meanness property for some standard graphs.

\section{Introduction and Preliminaries}

All through this paper, by a graph we mean a simple, undirected, and finite graph. For documentations and wording, we follow [1-5]. For a point by point review on graph labeling, we refer [6]. The line graph $L(G)$ of a graph $G$ is defined to have as its vertices the edges of $G$, with two being adjacent if the corresponding edges share a vertex in $G$. The graph $G^{\circ} S_{m}$ is obtained from $G$ by attaching $m$ pendant vertices to each vertex of $G$. Let $u_{\alpha}: 1 \leq \alpha \leq n$ and $v_{\beta}^{(\alpha)}: 1 \leq \beta \leq m+1$ be the nodes of path $P_{n}$ and $\alpha^{\text {th }}$ copy of the star graph $S_{m}$, respectively, then the graph $\left[P_{n} ; S_{m}\right]$ is obtained from $n$ copies of $S_{m}$ and the path $P_{n}$ by joining $u_{\alpha}$ with the central vertex $v_{1}^{(\alpha)}$ of the $\alpha^{\text {th }}$ copy of $S_{m}$ by means of an edge, for $1 \leq \alpha \leq n$. A graph obtained by subdividing edge of $G$ by a vertex is called subdivision graph $S(G)$ and a graph obtained from the path by replacing every edge of a path by a $C_{3}$ is called triangular snake graph $T_{n}$.

\section{Literature Survey}

The investigation of graceful labeling is characterized by Rosa in [7] and prime labeling is defined by Tout et al. in [8]. Somasundram and Ponraj introduced the mean labeling of graphs in [9]. Durai Baskar and Arockiaraj defined the F-harmonic mean labeling [10] and discussed its meanness for some standard graphs. The idea of F-geometric was presented by Durai Baskar et al. in [11] and $F$-root mean labeling was presented by Arockiaraj et al. in [12] and talked about its meanness of ladder graph in [13]. Vaidya and Barasara in [14] have discussed so many results on product cordial labeling. Vaidya and Lekha in [15] presented the idea of a bi-odd sequential labeling. The labeling of $L(2,1)$ in $[16]$ is researched by Prajapati and Patel. Rajesh Kannan et al. discussed the FCM labeling of graphs and its line graphs in [17]. Propelled by and crafted by such a large number of creators in the territory of graph labeling, we present another labeling called classical mean labeling. A classical mean of two positive integers need not be an integer in general. For the classical mean is to be an integer, we may use either flooring or ceiling function. In this paper, we consider only the flooring function of our discussion and try to analyze that the line graph operation preserves the classical meanness property for some standard graphs. The labeling is one of the well studied area in Graph Theory. So, we are interested in defining new labeling called classical mean labeling. A classical mean labeling is for getting more accuracy of all the edge labeling by using the average of four different types of means of the vertex labeling of the given graph. Recently, Muhiuddin et al. studied various related concepts on graphs (see [18-22]). 
Graph labeling assumes an essential job in different areas of the real world system. The concepts of classical mean labeling are utilized to demonstrate numerous kinds of processes and relations in biological, social, material physical, and data systems. It is a powerful tool that makes complicated patterns to be learned easily and conveniently in various fields. A static network can be represented as a specific kind of graph by connecting nodes in some topology, and labeling can be applied for automatic routing of data in a network. The graph can be cycle, path, circuit, walk, and connected which represent a fixed network. For each network, labeling is done with a constant which helps routing to automatically detect next node in the network. The classical mean labeling is used in fast communication in sensor networks for finding the more accuracy level of sensor units.

\section{Methodology}

A function $\chi$ is known as a classical mean labeling of a graph $G(V, E)$ with $p$ nodes and $q$ edges if $\chi: V(G) \longrightarrow\{1,2,3, \ldots, q+1\}$ is injective and the incited edge assignment function $\chi^{*}: E(G) \longrightarrow\{1,2,3, \ldots, q\}$ characterized as

$$
\begin{aligned}
\chi^{*}(u v)= & \frac{1}{4}\left(\frac{\chi(u)+\chi(v)}{2}+\sqrt{\chi(u) \chi(v)}+\frac{2 \chi(u) \chi(v)}{\chi(u)+\chi(v)}\right. \\
& \left.\left.+\sqrt{\frac{\chi(u)^{2}+\chi(v)^{2}}{2}}\right)\right\rfloor,
\end{aligned}
$$

for all $u v \in E(G)$, is bijective. From Figure 1, a graph that concedes a classical mean labeling is said to be classical mean graph.

As $q$ is the number of edges of the given graph, it cannot take a randomly large number so that such a labeling exists. However more than one classical mean labeling exists for the given graph. So, we show one among in the proof.

Here, it is found that the line graph operation preserves the classical meanness property for some standard graphs.

\section{Classical Meanness of Some Standard Graphs and Its Line Graph}

Theorem 1. Every path $P_{n}$ for $n \geq 1$ and its line graph $L\left(P_{n}\right)$ for $n \geq 2$ are classical mean graphs.

Proof. Develop a mapping $\chi$ from the vertex set of path to $\{1,2,3, \ldots, n\}$ by $\chi\left(v_{\alpha}\right)=\alpha$, for $1 \leq \alpha \leq n$, where $\left\{v_{\alpha}: 1 \leq \alpha \leq n\right\}$ be the nodes of the path. Therefore, for $1 \leq \alpha \leq n-1, \chi^{*}\left(v_{\alpha} v_{\alpha+1}\right)=\alpha$. Since $L\left(P_{n}\right)$ is again a path, $L\left(P_{n}\right)$ is also a classical mean graph. Hence, every path $P_{n}$ for $n \geq 1$ and its line graph $L\left(P_{n}\right)$ for $n \geq 2$ are classical mean graphs.

Theorem 2. Every cycle $C_{n}$ and its line graph $L\left(C_{n}\right)$ are classical mean graphs, for $n \geq 3$.

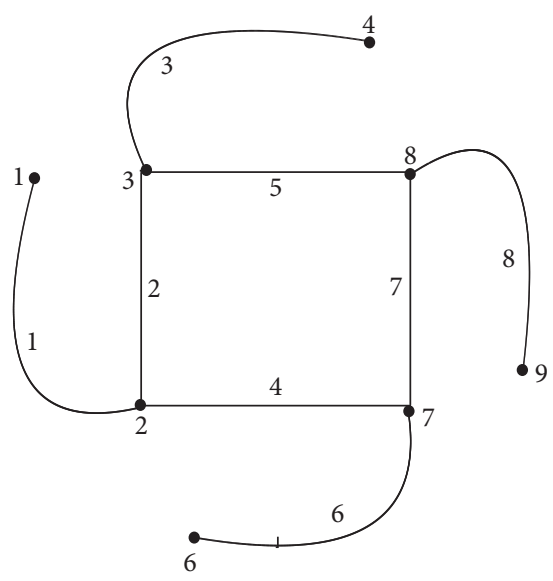

Figure 1: A classical mean labeling of $\mathrm{C}_{4}{ }^{\circ} \mathrm{S}_{1}$.

Proof. Develop a mapping $\chi$ from the vertex set of cycle to $\{1,2,3, \ldots, n+1\}$ by

$$
\chi\left(u_{\alpha}\right)= \begin{cases}-1+2 \alpha, & 1 \leq \alpha \leq\left\lfloor\frac{n}{2}\right\rfloor+1, \\ 2 n-2 \alpha+4, & \left\lfloor\frac{n}{2}\right\rfloor+2 \leq \alpha \leq n .\end{cases}
$$

Therefore,

$$
\chi^{*}\left(u_{\alpha} u_{1+\alpha}\right)= \begin{cases}2 \alpha-1, & 1 \leq \alpha \leq\left\lfloor\frac{n}{2}\right\rfloor, \\ n, & \alpha=\left\lfloor\frac{n}{2}\right\rfloor+1, \\ 2 n-2 \alpha+2, & \left\lfloor\frac{n}{2}\right\rfloor+2 \leq \alpha \leq-1+n \text { and }\end{cases}
$$

Also, the graph $L\left(C_{n}\right)$ is again a cycle, which is given by Figure 2. Hence, every cycle $C_{n}$ and its line graph $L\left(C_{n}\right)$ are classical mean graphs, for $n \geq 3$.

\section{Classical Meanness of Graphs Obtained from Vertex Identification and Its Line Graph}

Theorem 3. . The graph $P_{n} \circ S_{m}$ and its line graph $L\left(P_{n} \circ S_{m}\right)$ are classical mean graphs, for $n \geq 1$ and $m \leq 2$.

Proof. Let $\left\{u_{\beta}^{\alpha}: 1 \leq \alpha \leq n\right.$ and $\left.1 \leq \beta \leq m\right\}$ be the pendant vertices at each $v_{\alpha}$.

Case i. $m=1$ in the graph $P_{n}{ }^{\circ} \mathrm{S}_{\mathrm{m}}$. Develop a mapping $\chi$ from the vertex set of $P_{n}{ }^{\circ} S_{1}$ to $\{1,2,3, \ldots, 2 n\}$ by

$$
\begin{aligned}
\chi\left(v_{\alpha}\right) & = \begin{cases}1, & \alpha=1, \\
2 \alpha, & 2 \leq \alpha \leq n \text { and },\end{cases} \\
\chi\left(u_{1}^{(\alpha)}\right) & = \begin{cases}2, & \alpha=1, \\
2 \alpha-1, & 2 \leq \alpha \leq n .\end{cases}
\end{aligned}
$$

Therefore, 


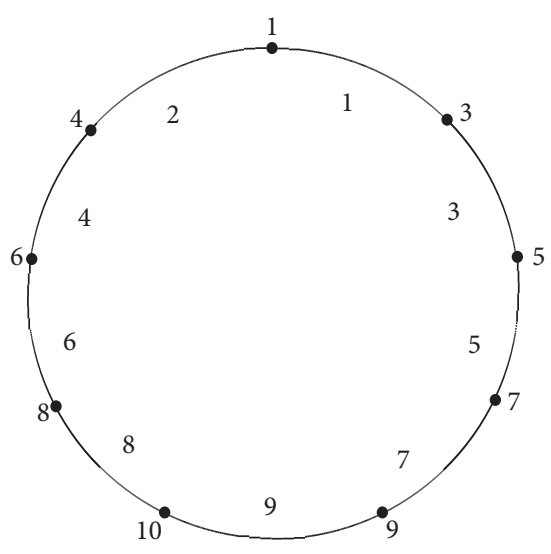

Figure 2: A classical mean labeling of $C_{9}$.

$$
\begin{aligned}
\chi^{*}\left(v_{\alpha+1} v_{\alpha}\right) & =2 \alpha, \text { for } 1 \leq \alpha \leq n-1 \text { and }, \\
\chi^{*}\left(u_{1}^{(\alpha) v_{\alpha}}\right) & =-1+2 \alpha, 1 \leq \alpha \leq n .
\end{aligned}
$$

Case ii. $m=2$ in the graph $P_{n}{ }^{\circ} \mathrm{S}_{\mathrm{m}}$.

Develop a mapping $\chi$ from the vertex set of $P_{n}{ }^{\circ} S_{2}$ to $\{1,2,3, \ldots, 3 n\}$ by

$$
\begin{gathered}
\chi\left(v_{\alpha}\right)=3 \alpha-1, \text { for } 1 \leq \alpha \leq n, \\
\chi\left(u_{1}^{(\alpha)}\right)=3 \alpha-2, \text { for } 1 \leq \alpha \leq n \text { and } \\
\chi\left(u_{2}^{(\alpha)}\right)=3 \alpha, \text { for } 1 \leq \alpha \leq n
\end{gathered}
$$

Therefore,

$$
\begin{aligned}
& \chi^{*}\left(v_{\alpha} v_{\alpha+1}\right)=3 \alpha, \text { for } 1 \leq \alpha \leq n-1, \\
& \chi^{*}\left(v_{\alpha} u_{1}^{(\alpha)}\right)=3 \alpha-2, \text { for } 1 \leq \alpha \leq n \text { and }, \\
& \chi^{*}\left(v_{\alpha} u_{2}^{(\alpha)}\right)=3 \alpha-1, \text { for } 1 \leq \alpha \leq n .
\end{aligned}
$$

Hence, a classical mean labeling of $P_{7} \circ \mathrm{S}_{1}$ and $P_{7} \circ \mathrm{S}_{2}$ is given by Figure 3.

Let $\quad V\left(L\left(P_{n}{ }^{\circ} \mathrm{S}_{1}\right)\right)=\left\{\mathrm{v}_{1}, \mathrm{v}_{2}, \mathrm{v}_{3}, \ldots, \mathrm{v}_{\mathrm{n}}, \mathrm{e}_{1}, \mathrm{e}_{2}, \mathrm{e}_{3}, \ldots\right.$, $\left.e_{n-1}\right\}$ and $E\left(L\left(P_{n}{ }^{\circ} \mathrm{S}_{1}\right)\right)=\left\{\mathrm{v}_{\alpha} \mathrm{e}_{\alpha}, \mathrm{e}_{\alpha} \mathrm{v}_{\alpha+1}: 1 \leq \alpha \leq \mathrm{n}-1\right\} \cup$ $\left\{e_{\alpha} e_{\alpha+1}: 1 \leq \alpha \leq n-2\right\}$.

Case iii. $m=1$ in the graph $L\left(P_{n}^{\circ} \mathrm{S}_{\mathrm{m}}\right)$.

Develop a mapping $\chi$ from the line graph of vertex set of $P_{n}{ }^{\circ} S_{1}$ to $\{1,2,3, \ldots, 3 n-3\}$ by

$$
\begin{aligned}
& \chi\left(v_{\alpha}\right)= \begin{cases}2 \alpha-1, & 1 \leq \alpha \leq 3, \\
3 \alpha-4, & 4 \leq \alpha \leq n \text { and },\end{cases} \\
& \chi\left(e_{\alpha}\right)= \begin{cases}2, & \alpha=1, \\
3 \alpha, & 2 \leq \alpha \leq n-1 .\end{cases}
\end{aligned}
$$

Therefore,

$$
\begin{aligned}
& \chi^{*}\left(v_{\alpha} e_{\alpha}\right)= \begin{cases}3 \alpha-2, & 1 \leq \alpha \leq 2, \\
3 \alpha-3, & 3 \leq \alpha \leq-1+n,\end{cases} \\
& \chi^{*}\left(e_{\alpha} v_{\alpha+1}\right)=3 \alpha-1, \quad \text { for } 1 \leq \alpha \leq n-1 \text { and, } \\
& \chi^{*}\left(e_{\alpha} e_{\alpha+1}\right)= \begin{cases}3, & \alpha=1, \\
3 \alpha+1, & 2 \leq \alpha \leq-2+n .\end{cases}
\end{aligned}
$$

Let $\quad V\left(L\left(P_{n}^{\circ}{ }^{\circ} \mathrm{S}_{2}\right)\right)=\left\{\mathrm{x}_{\alpha}: 1 \leq \alpha \leq \mathrm{n}-1\right\} \cup\left\{\mathrm{y}_{\alpha}, \mathrm{z}_{\alpha}: 1 \leq\right.$ $\alpha \leq n\}$ and $E\left(L\left(P_{n}^{\circ} \mathrm{S}_{2}\right)\right)=\left\{\mathrm{x}_{\alpha} \mathrm{z}_{\alpha}, \mathrm{x}_{\alpha} \mathrm{y}_{\alpha+1}, \mathrm{x}_{\alpha} \mathrm{z}_{\alpha+1}, x_{\alpha} y_{\alpha}\right.$ : $1 \leq \alpha \leq n-1\} \cup\left\{x_{\alpha} x_{\alpha+1}: 1 \leq \alpha \leq n-2\right\} \cup\left\{y_{\alpha} z_{\alpha}: 1 \leq \alpha \leq\right.$ $n\}$.

Case iv. $m=2$ in the graph $L\left(P_{n}{ }^{\circ} \mathrm{S}_{\mathrm{m}}\right)$. Develop a mapping $\chi$ from the line graph of vertex set of $P_{n}{ }^{\circ} S_{2}$ to $\{1,2,3, \ldots, 6 n-5\}$ by

$$
\begin{aligned}
& \chi\left(x_{\alpha}\right)= \begin{cases}5, & \alpha=1, \\
6 \alpha, & 2 \leq \alpha \leq n-1,\end{cases} \\
& \chi\left(y_{\alpha}\right)= \begin{cases}1, & \alpha=1, \\
6 \alpha-8, & 2 \leq \alpha \leq n \text { and },\end{cases} \\
& \chi\left(z_{\alpha}\right)= \begin{cases}6 \alpha-4, & 1 \leq \alpha \leq 2, \\
6 \alpha-5, & 3 \leq \alpha \leq n .\end{cases}
\end{aligned}
$$

Therefore,

$$
\begin{aligned}
& \chi^{*}\left(x_{\alpha} x_{\alpha+1}\right)=2+6 \alpha, \quad \text { for } 1 \leq \alpha \leq n-2 \text {, } \\
& \chi^{*}\left(x_{\alpha} y_{\alpha}\right)= \begin{cases}2, & \alpha=1, \\
6 \alpha-5, & 2 \leq \alpha \leq-1+n,\end{cases} \\
& \chi^{*}\left(x_{\alpha} z_{\alpha}\right)=6 \alpha-3, \quad \text { for } 1 \leq \alpha \leq n-1 \text {, } \\
& \chi^{*}\left(x_{\alpha} z_{\alpha+1}\right)=6 \alpha, \quad \text { for } 1 \leq \alpha \leq-1+n \text {, } \\
& \chi^{*}\left(x_{\alpha} y_{\alpha+1}\right)=6 \alpha-2, \quad \text { for } 1 \leq \alpha \leq-1+n \text { and, } \\
& \chi^{*}\left(y_{\alpha} z_{\alpha}\right)= \begin{cases}1, & \alpha=1, \\
6 \alpha-7, & 2 \leq \alpha \leq n .\end{cases}
\end{aligned}
$$

Hence, from Figure 4, the graph $P_{n}{ }^{\circ} S_{\mathrm{m}}$ and its line graph $L\left(P_{n}{ }^{\circ} \mathrm{S}_{\mathrm{m}}\right)$ are classical mean graphs, for $m \leq 2$ and $n \geq 1$.

Theorem 4. The graph $\left[P_{n} ; S_{m}\right]$ and its line graph $L\left(\left[P_{n} ; S_{m}\right]\right)$ are classical mean graphs, for $m \leq 2$ and $n \geq 1$.

Proof.

Case i. $m=1$ in the graph $\left[P_{n} ; S_{m}\right]$. Develop a mapping $\chi$ from the vertex set of $\left[P_{n} ; S_{1}\right]$ to $\{1,2,3, \ldots, 3 n\}$ by

$$
\begin{aligned}
\chi\left(u_{\alpha}\right) & = \begin{cases}3 \alpha, & \alpha \text { is odd and } 1 \leq \alpha \leq n, \\
3 \alpha-2, & \alpha \text { is even and } 1 \leq \alpha \leq n,\end{cases} \\
\chi\left(v_{1}^{(\alpha)}\right) & =3 \alpha-1, \quad 1 \leq \alpha \leq n \text { and, }
\end{aligned}
$$

Therefore, 

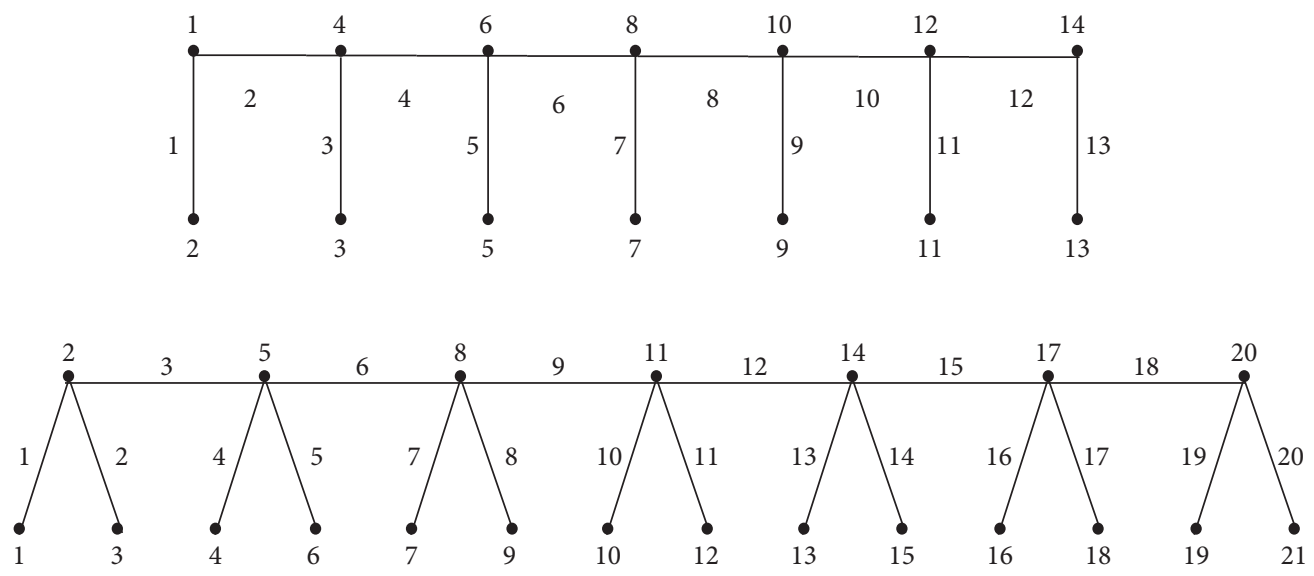

Figure 3: A classical mean labeling of $P_{7}^{\circ} \mathrm{S}_{1}$ and $P_{7}{ }^{\circ} \mathrm{S}_{2}$.
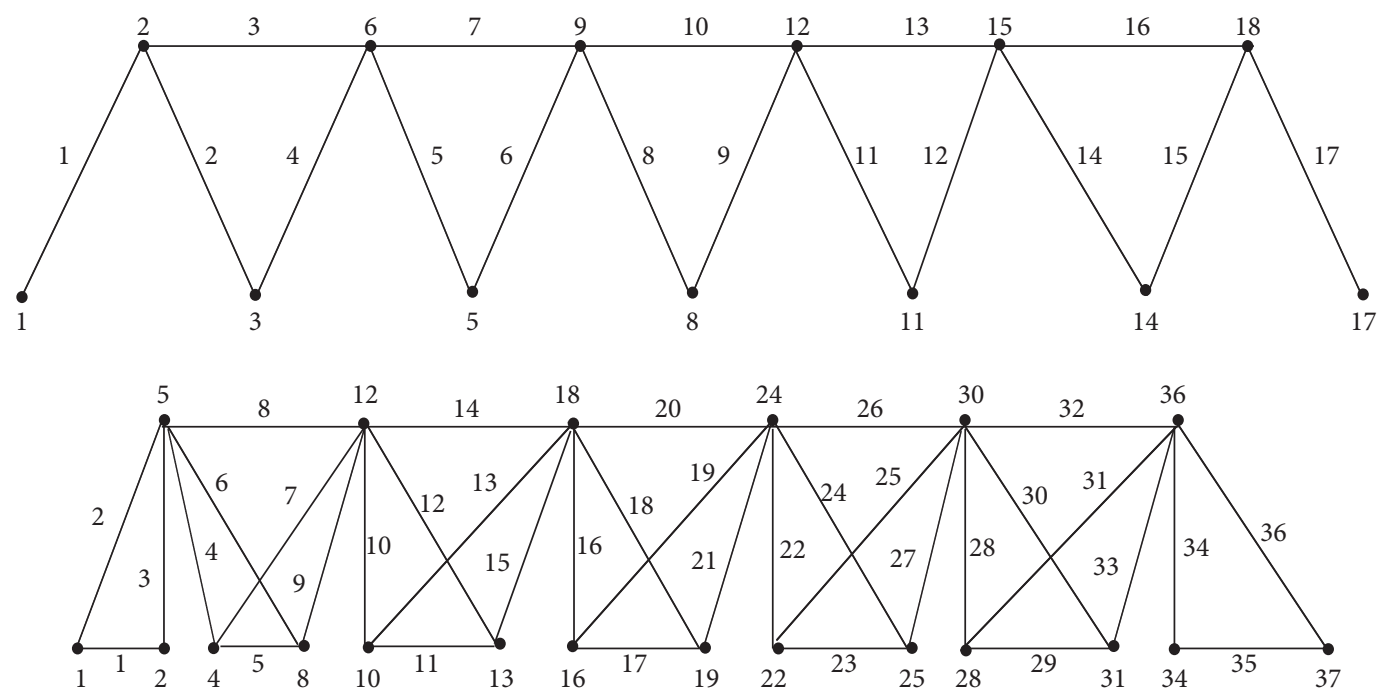

Figure 4: A classical mean labeling of $L\left(P_{7}^{\circ} \mathrm{S}_{1}\right)$ and $L\left(P_{7}^{\circ} \mathrm{S}_{2}\right)$.

$\chi^{*}\left(u_{\alpha} u_{\alpha+1}\right)=3 \alpha$, for $1 \leq \alpha \leq n-1$,

$\chi^{*}\left(u_{\alpha} v_{1}^{(\alpha)}\right)= \begin{cases}-1+3 \alpha, & \alpha \text { is odd and } 1 \leq \alpha \leq n, \\ -2+3 \alpha, & \alpha \text { is even and } 1 \leq \alpha \leq n \text { and }\end{cases}$

$\chi^{*}\left(v_{1}^{(\alpha)} v_{2}^{(\alpha)}\right)= \begin{cases}-2+3 \alpha, & \alpha \text { is odd and } 1 \leq \alpha \leq n, \\ -1+3 \alpha, & \alpha \text { is even and } 1 \leq \alpha \leq n .\end{cases}$

Case ii. $m=2$ in the graph $\left[P_{n} ; S_{m}\right]$. Develop a mapping $\chi$ from the vertex set of $\left[P_{n} ; S_{2}\right]$ to $\{1,2,3, \ldots, 4 n\}$ by

$$
\begin{aligned}
\chi\left(u_{\alpha}\right) & = \begin{cases}4 \alpha, & \alpha \text { is odd and } 1 \leq \alpha \leq n, \\
-2+4 \alpha, & \alpha \text { is even and } 1 \leq \alpha \leq n,\end{cases} \\
\chi\left(v_{1}^{(\alpha)}\right) & =4 \alpha-1, \quad 1 \leq \alpha \leq n, \\
\chi\left(v_{2}^{(\alpha)}\right) & = \begin{cases}1, & \alpha=1, \\
4 \alpha+1, & 2 \leq \alpha \leq n \text { and },\end{cases} \\
\chi\left(v_{3}^{(\alpha)}\right) & = \begin{cases}4 \alpha-2, & \alpha \text { is odd and } 1 \leq \alpha \leq n, \\
4 \alpha, & \alpha \text { is even and } 1 \leq \alpha \leq n .\end{cases}
\end{aligned}
$$

Therefore, 


$$
\begin{aligned}
& \chi^{*}\left(u_{\alpha} u_{\alpha+1}\right)=4 \alpha \text {, for } 1 \leq \alpha \leq n-1 \text {, } \\
& \chi^{*}\left(u_{\alpha} v_{1}^{(\alpha)}\right)= \begin{cases}-1+4 \alpha, & \alpha \text { is odd and } 1 \leq \alpha \leq n, \\
-2+4 \alpha, & \alpha \text { is even and } 1 \leq \alpha \leq n,\end{cases} \\
& \chi^{*}\left(v_{1}^{(\alpha)} v_{2}^{(\alpha)}\right)=-3+4 \alpha, \quad \text { for } 1 \leq \alpha \leq n-1, \\
& \chi^{*}\left(v_{1}^{(\alpha)} v_{3}^{(\alpha)}\right)= \begin{cases}-2+4 \alpha, & \alpha \text { is odd and } 1 \leq \alpha \leq n, \\
4 \alpha-1, & \alpha \text { is even and } 1 \leq \alpha \leq n .\end{cases}
\end{aligned}
$$

It is clearly seen that a classical mean labeling of $\left[P_{7} ; S_{1}\right]$ and $\left[P_{6} ; S_{2}\right]$ is given by Figure 5 .

Case iii. $m=1$ and $n \geq 2$ in the graph $L\left(\left[P_{n} ; S_{m}\right]\right)$. Develop a mapping $\chi$ from the line graph of vertex set of $\left[P_{n} ; S_{1}\right]$ to $\{1,2,3, \ldots, 4 n-3\}$ by

$$
\begin{aligned}
\chi\left(u_{\alpha}\right) & =4 \alpha, \quad \text { for } 1 \leq \alpha \leq n-1, \\
\chi\left(v_{1}^{(\alpha)}\right) & = \begin{cases}2, & \alpha=1, \\
4 \alpha-3, & 2 \leq \alpha \leq n \text { and, }\end{cases} \\
\chi\left(v_{2}^{(\alpha)}\right) & = \begin{cases}1, & \alpha=1, \\
4 \alpha-5, & 2 \leq \alpha \leq n .\end{cases}
\end{aligned}
$$

Therefore,

$$
\begin{aligned}
\chi^{*}\left(u_{\alpha} u_{\alpha+1}\right) & =4 \alpha+1, \quad \text { for } 1 \leq \alpha \leq n-1, \\
\chi^{*}\left(u_{\alpha} v_{1}^{(\alpha+1)}\right) & =4 \alpha, \quad \text { for } 1 \leq \alpha \leq n-1, \\
\chi^{*}\left(u_{\alpha} v_{1}^{(\alpha)}\right) & =4 \alpha-2, \quad \text { for } 1 \leq \alpha \leq n-1 \text { and }, \\
\chi^{*}\left(v_{1}^{(\alpha)} v_{2}^{(\alpha)}\right) & = \begin{cases}1, & \alpha=1, \\
4 \alpha-5, & 2 \leq \alpha \leq n .\end{cases}
\end{aligned}
$$

Case iv. $m=1$ and $n=1$ in the graph $L\left(\left[P_{n} ; S_{m}\right]\right)$. For $n=1$, the graph $L\left(\left[P_{n} ; S_{1}\right]\right)$ is a path and by Theorem 1 , the result follows.

Case v. $m=2$ and $n \geq 2$ in the graph $L\left(\left[P_{n} ; S_{m}\right]\right)$. Develop a mapping $\chi$ from the line graph of vertex set of $\left[P_{n} ; S_{2}\right]$ to $\{1,2,3, \ldots, 5 n-3\}$ by

$$
\begin{aligned}
& \chi\left(u_{\alpha}\right)= \begin{cases}-4+8 \alpha, & 1 \leq \alpha \leq 2, \\
5 \alpha, & \alpha \text { is odd } \text { and } 3 \leq \alpha \leq n, \\
1+5 \alpha, & \alpha \text { is even and } 1 \leq \alpha \leq n,\end{cases} \\
& \chi\left(v_{1}^{(\alpha)}\right)= \begin{cases}3 \alpha, & 1 \leq \alpha \leq 2, \\
11, & \alpha=3, \\
5 \alpha-3, & 4 \leq \alpha \leq n \text { and } \alpha \text { is odd, } \\
5 \alpha-4, & 4 \leq \alpha \leq n \text { and } \alpha \text { is even, }\end{cases} \\
& \chi\left(v_{2}^{(\alpha)}\right)= \begin{cases}4 \alpha-3, & 1 \leq \alpha \leq 2, \\
5 \alpha-7, & 3 \leq \alpha \leq n \text { and } \alpha \text { is odd, } \\
-6+5 \alpha, & 3 \leq \alpha \leq n \text { and } \alpha \text { is even and },\end{cases} \\
& \chi\left(v_{3}^{(\alpha)}\right)= \begin{cases}2, & \alpha=1, \\
-5+5 \alpha, & \alpha \text { is odd and } 2 \leq \alpha \leq n, \\
-3+5 \alpha, & \alpha \text { is even and } 2 \leq \alpha \leq n .\end{cases}
\end{aligned}
$$

Therefore,

$$
\begin{aligned}
& \chi^{*}\left(u_{\alpha} u_{\alpha+1}\right)= \begin{cases}6 \alpha+1, & 1 \leq \alpha \leq 2, \\
5 \alpha+2, & 3 \leq \alpha \leq n-2,\end{cases} \\
& \chi^{*}\left(u_{\alpha} v_{1}^{(\alpha)}\right)= \begin{cases}3, & \alpha=1, \\
4 \alpha, & 2 \leq \alpha \leq 3, \\
5 \alpha-2, & 4 \leq \alpha \leq n-1,\end{cases} \\
& \chi^{*}\left(u_{\alpha} v_{1}^{(\alpha+1)}\right)= \begin{cases}4, & \alpha=1, \\
5 \alpha, & \alpha \text { is odd and } 2 \leq \alpha \leq n, \\
5 \alpha+1, & \alpha \text { is even and } 2 \leq \alpha \leq n,\end{cases} \\
& \chi^{*}\left(v_{1}^{(\alpha)} v_{2}^{(\alpha)}\right)= \begin{cases}4 \alpha-3, & 1 \leq \alpha \leq 2, \\
5 \alpha-6, & 3 \leq \alpha \leq n \text { and },\end{cases} \\
& \chi^{*}\left(v_{1}^{(\alpha)} v_{3}^{(\alpha)}\right)= \begin{cases}2, & \alpha=1, \\
5 \alpha-5, & \alpha \text { is odd and } 2 \leq \alpha \leq n, \\
5 \alpha-4, & \alpha \text { is even and } 2 \leq \alpha \leq n\end{cases}
\end{aligned}
$$

Case vi. $m=2$ and $n=1$ in the graph $L\left(\left[P_{n} ; S_{m}\right]\right)$.

For $n=1$, the graph $L\left(\left[P_{1} ; S_{2}\right]\right)$ is $C_{3}$ and by Theorem 2, the result follows.

Hence, the graph $\left[P_{n} ; S_{m}\right]$ for $m \leq 2$ and $n \geq 1$ and its line graph $L\left(\left[P_{n} ; S_{m}\right]\right)$ for $n \geq 1$ are classical mean graphs given by Figure 6.

\section{Classical Meanness of Graphs Obtained from Other Graph Operations and Its Line Graph}

Theorem 5. . For $n \geq 2, M\left(P_{n}\right)$ and its line graph $L\left(M\left(P_{n}\right)\right)$ are classical mean graphs.

Proof. Since $M\left(P_{n}\right)$ is a graph $L\left(P_{n}{ }^{\circ} S_{1}\right)$, for $n \geq 2$ and by Theorem 3, the result follows. Let $V(L(M$ $\left.\left.\left(P_{n}\right)\right)\right)=\left\{x_{\alpha}: 1 \leq \alpha \leq n+1\right\} \cup\left\{x_{\alpha}^{\prime}: 1 \leq \alpha \leq n-3\right\} \cup\left\{y_{\alpha}: 1 \leq \alpha\right.$ $\leq n-2\}$ and $E\left(L\left(M\left(P_{n}\right)\right)\right)=\left\{y_{\alpha} y_{\alpha+1}, x_{\alpha}^{\prime} x_{\alpha+3}, x_{\alpha}^{\prime} y_{\alpha+1}\right.$, $\left.y_{\alpha} x_{\alpha}^{\prime}: 1 \leq \alpha \leq n-3\right\} \cup\left\{y_{\alpha} x_{\alpha+2}: 1 \leq \alpha \leq n-2\right\} \cup\left\{y_{\alpha} x_{\alpha+1}: 2 \leq\right.$ $\alpha \leq n-2\} \cup\left\{x_{\alpha} x_{\alpha+1}, x_{\alpha} y_{1}: 1 \leq \alpha \leq 2\right\} \cup\left\{y_{n-2} x_{n+1}, x_{n} x_{n+1}\right\}$.

Case i. $n \geq 3$ in the graph $L\left(M\left(P_{n}\right)\right)$. Develop a mapping $\chi$ from the line graph of vertex set of $M\left(P_{n}\right)$ to $\{1,2,3, \ldots, 7 n-13\}$ by

$$
\begin{aligned}
& \chi\left(x_{\alpha}\right)= \begin{cases}\alpha, & 1 \leq \alpha \leq 2, \\
-14+7 \alpha, & 3 \leq \alpha \leq n, \\
-13+7 n, & \alpha=n+1,\end{cases} \\
& \chi\left(x_{\alpha}^{\prime}\right)=2+7 \alpha, \quad \text { for } 1 \leq \alpha \leq n-3 \text { and } \\
& \chi\left(y_{\alpha}\right)=-2+7 \alpha, \quad \text { for } 1 \leq \alpha \leq n-2 .
\end{aligned}
$$

Therefore, 

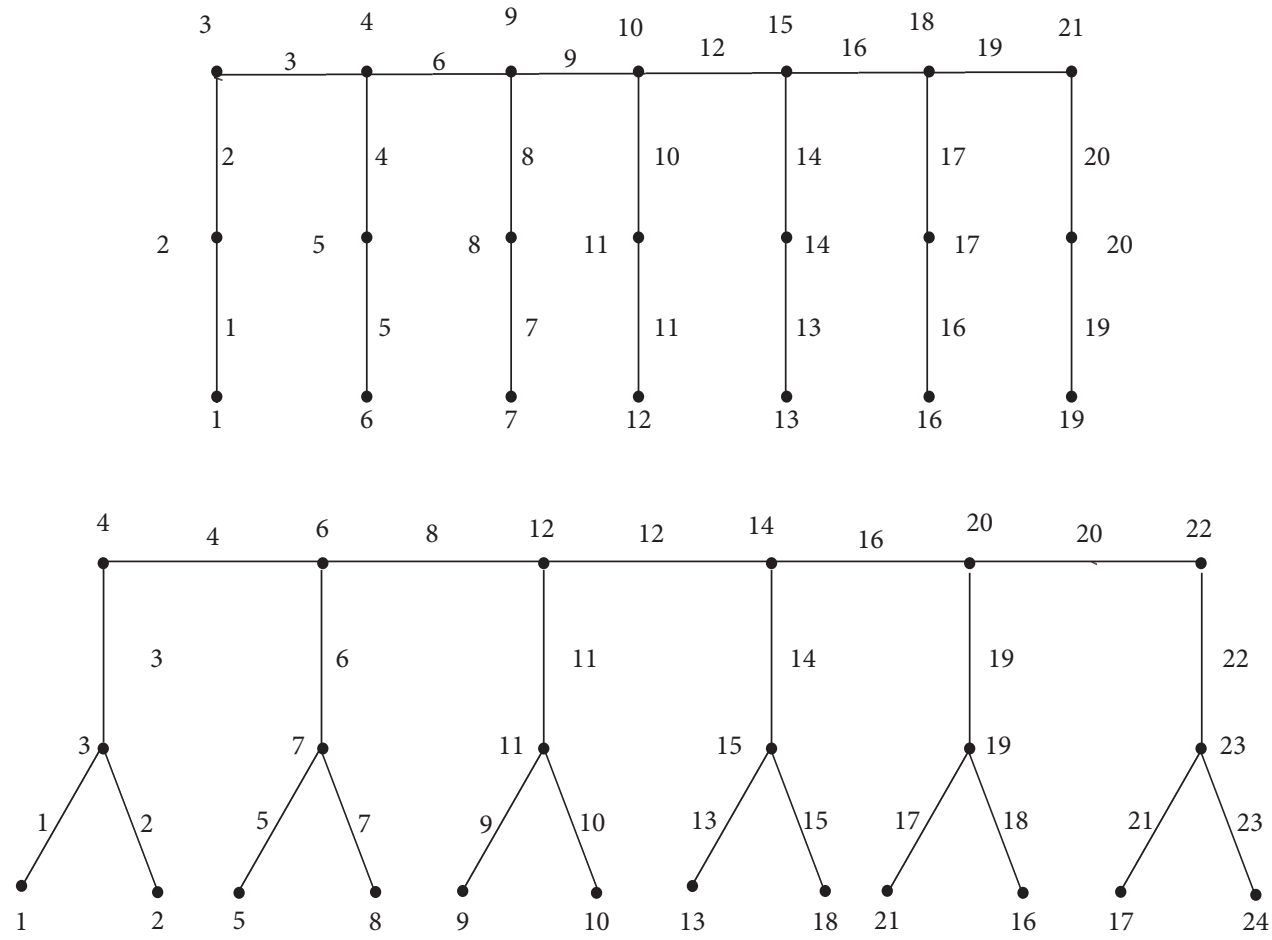

Figure 5: A classical mean labeling of $\left[P_{7} ; S_{1}\right]$ and $\left[P_{6} ; S_{2}\right]$.
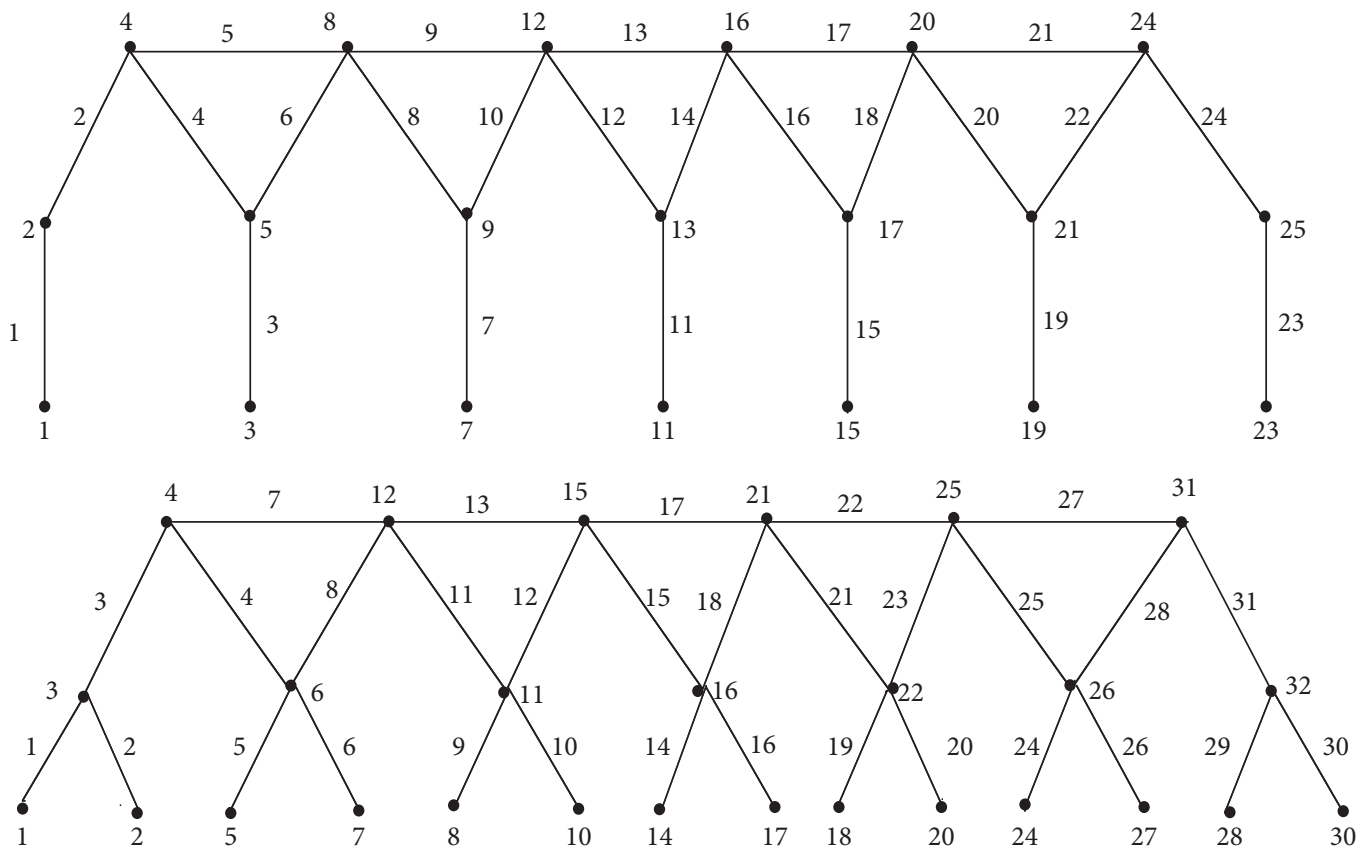

FIgURe 6: A classical mean labeling of $L\left(\left[P_{7} ; S_{1}\right]\right)$ and $L\left(\left[P_{7} ; S_{2}\right]\right)$. 


$$
\begin{aligned}
& \chi^{*}\left(y_{\alpha} y_{\alpha+1}\right)=7 \alpha+1, \quad \text { for } 1 \leq \alpha \leq n-3 \text {, } \\
& \chi^{*}\left(x_{\alpha}^{\prime} x_{\alpha+3}\right)=7 \alpha+4, \quad \text { for } 1 \leq \alpha \leq n-3 \text {, } \\
& \chi^{*}\left(x_{\alpha}^{\prime} y_{\alpha+1}\right)=7 \alpha+3, \quad \text { for } 1 \leq \alpha \leq n-3 \text {, } \\
& \chi^{*}\left(y_{\alpha} x_{\alpha}^{\prime}\right)=7 \alpha-1, \quad \text { for } 1 \leq \alpha \leq n-3 \text {, } \\
& \chi^{*}\left(y_{\alpha} x_{\alpha+2}\right)=7 \alpha-2, \quad \text { for } 1 \leq \alpha \leq n-2 \text {, } \\
& \chi^{*}\left(y_{\alpha} x_{\alpha+1}\right)= \begin{cases}3, & \alpha=1, \\
7 \alpha-5, & 2 \leq \alpha \leq n-2,\end{cases} \\
& \chi^{*}\left(x_{\alpha} x_{\alpha+1}\right)=3 \alpha-2, \quad \text { for } 1 \leq \alpha \leq 2 \text {, } \\
& \chi^{*}\left(x_{\alpha} y_{1}\right)=\alpha+1, \quad \text { for } 1 \leq \alpha \leq 2, \\
& \chi^{*}\left(y_{n-2} x_{n+1}\right)=7 n-15 \text { and } \\
& \chi^{*}\left(x_{n} x_{n+1}\right)=-14+7 n \text {. }
\end{aligned}
$$

Case ii. $n=2$ in the graph $L\left(M\left(P_{n}\right)\right)$. For $n=2$, the graph $L\left(M\left(P_{n}\right)\right)$ is $P_{2}$ and by Theorem 1 , the result follows. Hence, from Figure 7 , for $n \geq 2$, the graph $M\left(P_{n}\right)$ and its line graph $L\left(M\left(P_{n}\right)\right)$ are classical mean graphs.

Theorem 6. The graph $S\left(P_{n}{ }^{\circ} K_{1}\right)$ and its line graph $L\left(S\left(P_{n}{ }^{\circ} K_{1}\right)\right)$ are classical mean graphs, for $n \geq 1$.

Proof. Let $V\left(S\left(P_{n}{ }^{\circ} \mathrm{K}_{1}\right)\right)=\left\{\mathrm{u}_{\alpha}, \mathrm{v}_{\alpha}, \mathrm{x}_{\alpha}, \mathrm{y}_{\beta}: 1 \leq \alpha \leq \mathrm{n}, 1 \leq\right.$ $\beta \leq n-1\}$ and $E\left(S\left(P_{n}{ }^{\circ} \mathrm{K}_{1}\right)\right)=\left\{\mathrm{u}_{\alpha} \mathrm{x}_{\alpha}, \mathrm{v}_{\alpha} \mathrm{x}_{\alpha}: 1 \leq \alpha \leq \mathrm{n}\right\} \cup$ $\left\{u_{\alpha} y_{\alpha}, y_{\alpha} u_{\alpha+1}: 1 \leq \alpha \leq n-1\right\}$. Develop a mapping $\chi$ from the vertex set of $S\left(P_{n}{ }^{\circ} \mathrm{K}_{1}\right)$ to $\{1,2,3, \ldots, 4 n-1\}$ by

$$
\begin{aligned}
& \chi\left(u_{\alpha}\right)= \begin{cases}3 \alpha, & 1 \leq \alpha \leq 2, \\
4 \alpha-1, & 3 \leq \alpha \leq n,\end{cases} \\
& \chi\left(y_{\alpha}\right)= \begin{cases}4, & \alpha=1, \\
4 \alpha+1, & 2 \leq \alpha \leq n-1,\end{cases} \\
& \chi\left(x_{\alpha}\right)= \begin{cases}5 \alpha-3, & 1 \leq \alpha \leq 2, \\
4 \alpha-2, & 3 \leq \alpha \leq n \text { and }\end{cases} \\
& \chi\left(v_{\alpha}\right)= \begin{cases}4 \alpha-3, & 1 \leq \alpha \leq 2, \\
4 \alpha-4, & 3 \leq \alpha \leq n .\end{cases}
\end{aligned}
$$

Therefore,

$$
\begin{aligned}
& \chi^{*}\left(u_{\alpha} y_{\alpha}\right)=-1+4 \alpha, \quad 1 \leq \alpha \leq n-1, \\
& \chi^{*}\left(y_{\alpha} u_{\alpha+1}\right)= \begin{cases}4, & \alpha=1, \\
1+4 \alpha, & 2 \leq \alpha \leq n-1,\end{cases} \\
& \chi^{*}\left(u_{\alpha} x_{\alpha}\right)= \begin{cases}2, & \alpha=1, \\
4 \alpha-2, & 2 \leq \alpha \leq n \text { and, }\end{cases} \\
& \chi^{*}\left(v_{\alpha} x_{\alpha}\right)= \begin{cases}4 \alpha-3, & 1 \leq \alpha \leq 2, \\
4 \alpha-4, & 3 \leq \alpha \leq n .\end{cases}
\end{aligned}
$$

Let $\quad V\left(L\left(S\left(P_{n}{ }^{\circ} \mathrm{K}_{1}\right)\right)\right)=\left\{\mathrm{u}_{\alpha}, \mathrm{u}_{\beta}^{\prime}, \mathrm{v}_{\alpha}, \mathrm{w}_{\alpha}: 1 \leq \alpha \leq \mathrm{n}, 1 \leq\right.$ $\beta \leq n-2\}$ and $E\left(L\left(S\left(P_{n}{ }^{\circ} \mathrm{K}_{1}\right)\right)\right)=\left\{\mathrm{u}_{\alpha} \mathrm{v}_{\alpha}, \mathrm{v}_{\alpha} \mathrm{w}_{\alpha}: 1 \leq \alpha \leq n\right\} \cup$ $\left\{u_{\alpha}^{\prime} v_{\alpha+1}: 1 \leq \alpha \leq n-2\right\} \cup\left\{u_{\alpha} u_{\alpha-1}^{\prime}: 2 \leq \alpha \leq n-1\right\} \cup\left\{u_{\alpha}^{\prime} u_{\alpha+2}:\right.$ $1 \leq \alpha \leq n-2\} \cup u_{1} u_{2}$.
Case i. $n \geq 3$ in the graph $L\left(S\left(P_{n}{ }^{\circ} \mathrm{K}_{1}\right)\right)$. Develop a mapping $\chi$ from the line graph of vertex set of $S\left(P_{n}{ }^{\circ} K_{1}\right)$ to $\{1,2,3, \ldots, 5 n-4\}$ by

$$
\begin{aligned}
& \chi\left(u_{\alpha}\right)= \begin{cases}3, & \alpha=1, \\
5 \alpha-6, & 2 \leq \alpha \leq n-1, \\
5 n-4, & \alpha=n,\end{cases} \\
& \chi\left(u_{\alpha}^{\prime}\right)=5 \alpha+3, \quad \text { for } 1 \leq \alpha \leq n-2 \text {, } \\
& \chi\left(v_{\alpha}\right)= \begin{cases}2, & \alpha=1, \\
5 \alpha-4, & 2 \leq \alpha \leq n-1, \\
5 \alpha-5, & \alpha=n \text { and },\end{cases} \\
& \chi\left(w_{\alpha}\right)= \begin{cases}1, & \alpha=1, \\
5 \alpha, & 2 \leq \alpha \leq n-2, \\
5 n-6, & \alpha=n-1, \\
5 n-8, & \alpha=n .\end{cases}
\end{aligned}
$$

Therefore,

$$
\begin{aligned}
& \chi^{*}\left(u_{\alpha} v_{\alpha}\right)= \begin{cases}2, & \alpha=1 \\
5 \alpha-6, & 2 \leq \alpha \leq n-1, \\
5 n-5, & \alpha=n,\end{cases} \\
& \chi^{*}\left(v_{\alpha} w_{\alpha}\right)= \begin{cases}1, & \alpha=1 \\
5 \alpha-3, & 2 \leq \alpha \leq n-1 \\
5 n-7, & \alpha=n,\end{cases} \\
& \chi^{*}\left(u_{\alpha}^{\prime} v_{\alpha+1}\right)= \begin{cases}6, & \alpha=1 \\
5 \alpha+1, & 2 \leq \alpha \leq n-2,\end{cases} \\
& \chi^{*}\left(u_{\alpha} u_{\alpha-1}^{\prime}\right)=5 \alpha, \quad \text { for } 2 \leq \alpha \leq n-1 \text {, } \\
& \chi^{*}\left(u_{\alpha}^{\prime} u_{\alpha+2}\right)= \begin{cases}5 \alpha+3, & 1 \leq \alpha \leq n-3 \\
6 \alpha-1, & \alpha=n-2 \text { and, }\end{cases} \\
& \chi^{*}\left(u_{1} u_{2}\right)=3
\end{aligned}
$$

Case ii. $1 \leq n \leq 2$ in the graph $L\left(S\left(P_{n}{ }^{\circ} K_{1}\right)\right)$. For $1 \leq n \leq 2$, the graph $L\left(S\left(P_{n}{ }^{\circ} K_{1}\right)\right)$ is a path and by Theorem 1, the result follows. Hence, from Figure 9, the graph $S\left(P_{n}{ }^{\circ} \mathrm{K}_{1}\right)$ and its line graph $L\left(S\left(P_{n}{ }^{\circ} \mathrm{K}_{1}\right)\right)$ are classical mean graphs, for $n \geq 1$.

Theorem 7. The triangular snake $T_{n}$ and its line graph $L\left(T_{n}\right)$ are classical mean graphs, for $n \geq 2$.

Proof. Let $V\left(T_{n}\right)=\left\{u_{\alpha}: 1 \leq \alpha \leq n-1\right\} \cup\left\{v_{\alpha}^{\prime}: 1 \leq \alpha \leq n\right\}$ and $E\left(T_{n}\right)=\left\{u_{\alpha} v_{\alpha}, u_{\alpha} v_{\alpha+1}, v_{\alpha} v_{\alpha+1}: 1 \leq \alpha \leq n-1\right\}$. Develop a mapping $\chi$ from the vertex set of $T_{n}$ to $\{1,2,3, \ldots, 3 n-2\}$ by

$$
\begin{aligned}
& \chi\left(u_{\alpha}\right)=3 \alpha, \quad 1 \leq \alpha \leq n-1 \text { and } \\
& \chi\left(v_{\alpha}\right)=-2+3 \alpha, \quad 1 \leq \alpha \leq n .
\end{aligned}
$$

Therefore, 


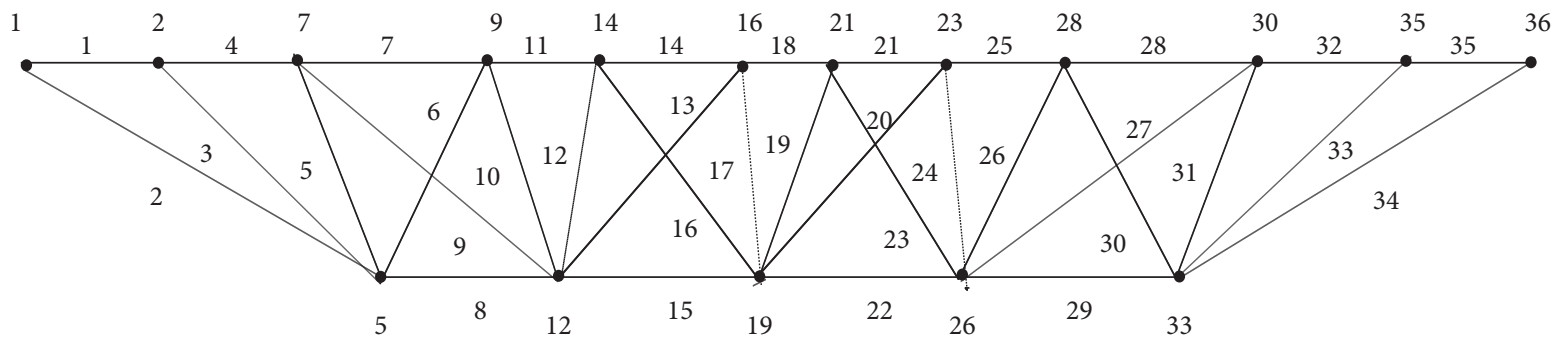

Figure 7: A classical mean labeling of $L\left(M\left(P_{7}\right)\right)$.

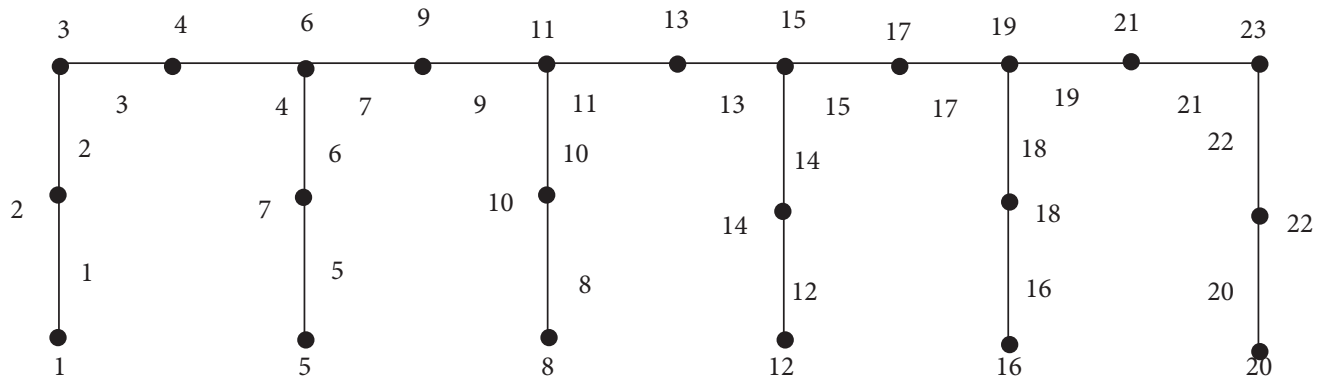

Figure 8: A classical mean labeling of $S\left(P_{6}{ }^{\circ} \mathrm{K}_{1}\right)$. Therefore, a classical mean labeling of $S\left(P_{6}{ }^{\circ} \mathrm{K}_{1}\right)$ is given by Figure 8 .

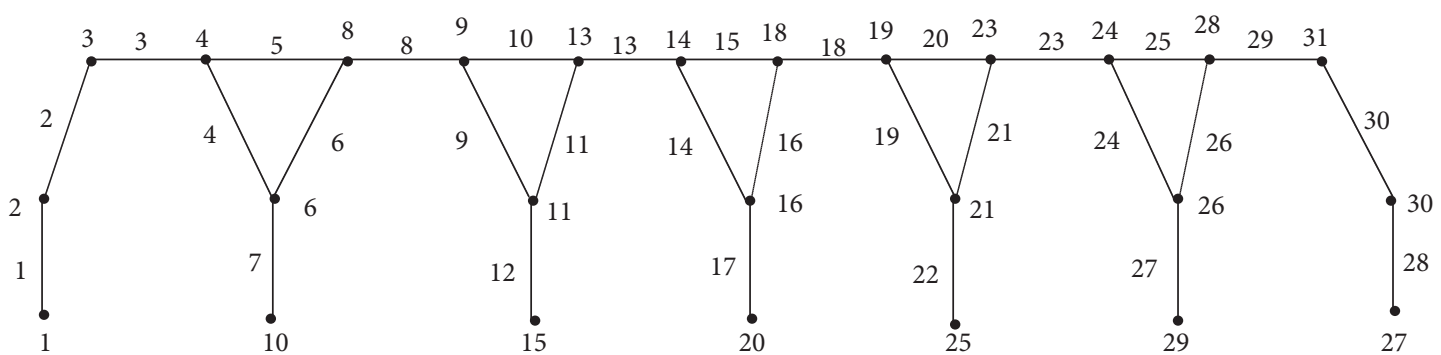

Figure 9: A classical mean labeling of $L\left(S\left(P_{7}{ }^{\circ} \mathrm{K}_{1}\right)\right)$.

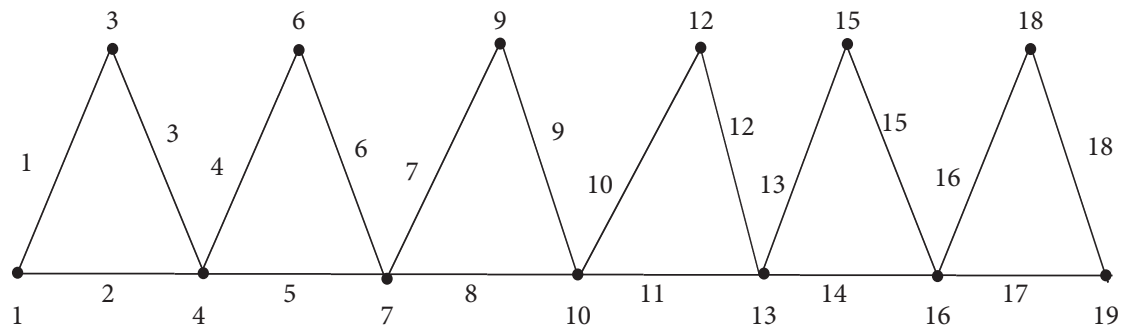

FIgURE 10: A classical mean labeling labeling of $T_{7}$.

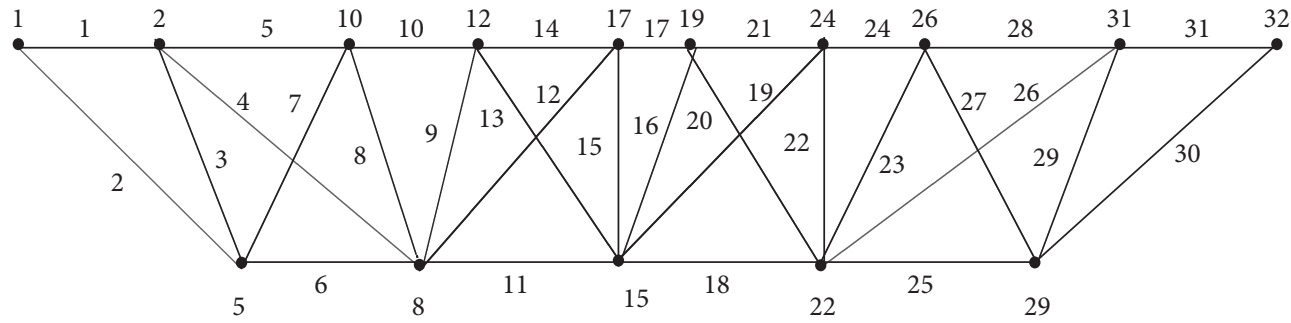

Figure 11: A classical mean labeling labeling of $L\left(T_{6}\right)$. 


$$
\begin{aligned}
\chi^{*}\left(u_{\alpha} v_{\alpha}\right) & =3 \alpha-2, \quad 1 \leq \alpha \leq n-1, \\
\chi^{*}\left(u_{\alpha} v_{\alpha+1}\right) & =3 \alpha, \quad 1 \leq \alpha \leq n-1 \text { and } \\
\chi^{*}\left(v_{\alpha} v_{\alpha+1}\right) & =-1+3 \alpha, \quad 1 \leq \alpha \leq n-1 .
\end{aligned}
$$

Hence, a classical mean labeling of $T_{7}$ is given by Figure 10.

Let $\quad V\left(L\left(T_{n}\right)\right)=\left\{x_{\alpha}: 1 \leq \alpha \leq n\right\} \cup\left\{x_{\alpha}^{\prime}: 1 \leq \alpha \leq n-2\right\} \cup$ $\left\{y_{\alpha}^{\prime}: 1 \leq \alpha \leq n-1\right\}$ and $E\left(L\left(T_{n}\right)\right)=\left\{y_{\alpha} y_{\alpha+1}, x_{\alpha} x_{\alpha-1}^{\prime}, \quad x_{\alpha^{\prime}}\right.$ $\left.x_{\alpha+2}: 1 \leq \alpha \leq n-2\right\} \cup\left\{y_{\alpha} x_{\alpha+1}, \quad y_{\alpha} x_{\alpha-1}^{\prime}, x_{\alpha} y_{\alpha}: 1 \leq \alpha \leq n-1\right\}$ $\cup\left\{x_{1} x_{2}, x_{2} x_{1}^{\prime}\right\}$.

Case i. $n \geq 3$ in the graph $L\left(T_{n}\right)$. Develop a mapping $\chi$ from the line graph of $T_{n}$ to $\{1,2,3, \ldots, 7 n-10\}$ by

$$
\begin{aligned}
& \chi\left(x_{\alpha}\right)= \begin{cases}\alpha, & 1 \leq \alpha \leq 2, \\
11, & \alpha=3 \text { and } \alpha=n, \\
12, & \alpha \neq 3 \text { and } \alpha=n, \\
7 \alpha-9, & 4 \leq \alpha \leq n-1, \\
7 \alpha-10, & \alpha=n,\end{cases} \\
& \chi\left(x_{\alpha}^{\prime}\right)=7 \alpha+3, \text { for } 1 \leq \alpha \leq n-2 \text { and } \\
& \chi\left(y_{\alpha}\right)= \begin{cases}5, & \alpha=1 . \\
7 \alpha-6, & 2 \leq \alpha \leq n-1 .\end{cases}
\end{aligned}
$$

Therefore,

$$
\begin{aligned}
& \chi^{*}\left(y_{\alpha} y_{\alpha+1}\right)= \begin{cases}6, & \alpha=1, \\
7 \alpha-3, & 2 \leq \alpha \leq n-2,\end{cases} \\
& \chi^{*}\left(y_{\alpha} x_{\alpha+1}\right)= \begin{cases}3, & \alpha=1, \\
7 \alpha-5, & 2 \leq \alpha \leq n-1,\end{cases} \\
& \chi^{*}\left(x_{\alpha} x_{\alpha-1}^{\prime}\right)= \begin{cases}5, & i=2, \\
7 \alpha-7, & 3 \leq \alpha \leq n-2,\end{cases} \\
& \chi^{*}\left(y_{\alpha} x_{\alpha-1}^{\prime}\right)=-6+7 \alpha, \quad \text { for } 2 \leq \alpha \leq n-1, \\
& \chi^{*}\left(x_{\alpha} y_{\alpha}\right)= \begin{cases}2 \alpha, & 1 \leq \alpha \leq 2, \\
-8+7 \alpha, & 3 \leq \alpha \leq n-1,\end{cases} \\
& \chi^{*}\left(x_{\alpha}^{\prime} x_{\alpha+2}\right)= \begin{cases}10, & \alpha=1, \\
7 \alpha+3, & 2 \leq \alpha \leq n-2,\end{cases} \\
& \chi^{*}\left(x_{1} x_{2}\right)=1 \text { and } \chi^{*}\left(x_{2} x_{1}^{\prime}\right)=5 \text {. }
\end{aligned}
$$

Case ii. $n=2$ in the graph $L\left(T_{n}\right)$. For $n=2$, the graph $L\left(T_{n}\right)$ is a cycle $C_{3}$ and by Theorem 2 , the result follows. Hence, from Figure 11, the triangular snake $T_{n}$ and its line graph $L\left(T_{n}\right)$ are classical mean graphs, for $n \geq 2$.

\section{Conclusion}

In this paper, it is found that the line graph operation preserves the classical meanness property for some standard graphs. Further investigation can be done to analyze the preservation of the classical meanness property by the line graph operation for other graphs.

\section{Data Availability}

No data were used to support the study.

\section{Conflicts of Interest}

The authors declare that they have no conflicts of interest.

\section{References}

[1] C. Berge, Theorie des Graphes et Ses Applications, Dunod, Paris, France, 1958.

[2] N. L. Biggs, E. K. Lloyd, and R. J. Wilson, Graph Theory, pp. 1736-1936, Clarendon Press, Oxford, UK, 1976.

[3] F. Harary, Graph Theory, Narosa Publishing House, New Delhi, India, 1988.

[4] O. Ore, Theory of Graphs, American Mathematical Society Colloquium Publications, Providence, RI, USA, 1962.

[5] R. Wilson, Four Colors Suffice: How the Map Problem Was Solved, Princeton University Press, Princeton, NJ, USA, 2002.

[6] J. A. Gallian, "A dynamic Survey of graph labeling," The Electronic Journal of Combinatorics, vol. 17, p. \#DS6, 2017.

[7] A. Rosa, "On certain valuations of the vertices of a graph," 1966.

[8] R. Tout, A. N. Dabboucy, and K. Howalla, "Prime labeling of graphs," National Academy Science Letters, vol. 5, pp. 365368, 1982.

[9] S. Somasundaram and R. Ponraj, "Mean labelings of graphs," National Academy Science Letters, vol. 26, pp. 210-213, 2003.

[10] A. Durai Baskar and S. Arockiaraj, "-harmonic mean graphs," International Journal of Mathematical Archieve, vol. 5, no. 8, pp. 92-108, 2014.

[11] A. Durai Baskar, S. Arockiaraj, and B. Rajendran, "Geometric meanness of graphs obtained from paths," Utliltas Mathematica, vol. 101, pp. 45-68, 2016.

[12] S. Arockiaraj, A. Durai Baskar, and A. Rajesh, "Kannan, F-root square mean labeling of graphs obtained from paths," International Journal of Mathematical Combinatorics, vol. 2, pp. 92-104, 2017.

[13] S. Arockiaraj, A. Durai Baskar, and A. Rajesh Kannan, "F-root square mean labeling of some ladder related graphs," Global Journal of Pure and Applied Mathematics, vol. 13, no. 2, pp. 281-289, 2019.

[14] S. K. Vaidya and C. M. Barasara, "Product cordial labeling of line graph of some graphs," Kragujevac Journal of Mathematics, vol. 40, no. 2, pp. 290-297, 2016.

[15] S. K. Vaidya and L. Bijukumar, "Some new perspectives on odd sequential graphs," International Journal of Mathematics and Soft Computing, vol. 4, no. 1, pp. 93-101, 2014.

[16] U. M. Prajapati and N. B. Patel, "Labeling of line graph of some graphs," Journal of Applied Science and Computations, vol. 6, no. 5, pp. 309-316, 2019.

[17] A. Rajesh Kannan, S. Arockiaraj, A. Durai Baskar, and P. Manivannan, "Further results on FCM labeling of some 
graphs and its line graphs," Advances in Mathematics: Scientific Journal, vol. 8, no. 3, pp. 797-805, 2019.

[18] S. S. Hussain, R. J. Hussain, and G. Muhiuddin, "Neutrosophic vague line graphs," Neutrosophic Sets and Systems, vol. 36, pp. 121-130, 2020.

[19] G. Muhiuddin, M. M. Takallo, Y. B. Jun, and R. A. Borzooei, "Cubic graphs and their application to a traffic flow problem," International Journal of Computational Intelligence Systems, vol. 13, no. 1, pp. 1265-1280, 2020.

[20] T. Pramanik, G. Muhiuddin, A. M. Alanazi, and M. Pal, "An Extension of fuzzy competition Graph and its uses in manufacturing industries," Mathematics, vol. 8, no. 6, p. 1008, 2020.

[21] H. Rashmanlou, G. Muhiuddin, Sk Amanathulla, F. Mofidnakhaei, and M. Pal, "A study on cubic graphs with novel application," Journal of Intelligent \& Fuzzy Systems, vol. 40, no. 1, pp. 89-101, 2021.

[22] S. Samanta, G. Muhiuddin, A. M. Alanazi, and K. Das, "Mathematical approach on representation of competitions: competition cluster hypergraphs," Mathematical Problems in Engineering, vol. 2020, Article ID 2517415, 10 pages, 2020. 